\title{
Effect of Dietary Patterns and, or Nutrition Strategies Reducing Risk in Adverse Maternal Health Occurrences during Pregnancy: A Systematic Review
}

Josephine Ansaa Larbi ( $\nabla$ anyomijosephine23@gmail.com )

University of GHANA, Nutrition and Food Science https://orcid.org/0000-0002-9977-4641

Systematic Review

Keywords: dietary patterns, nutrition strategies, maternal adverse health risks, adverse pregnancy and outcome

Posted Date: March 22nd, 2021

DOI: https://doi.org/10.21203/rs.3.rs-352358/v1

License: (9) This work is licensed under a Creative Commons Attribution 4.0 International License. Read Full License 


\section{Maternal and Child Health Journal \\ Effect of Dietary Patterns and, or Nutrition Strategies Reducing Risk in Adverse Maternal Health Occurrences during Pregnancy: A Systematic Review --Manuscript Draft--}

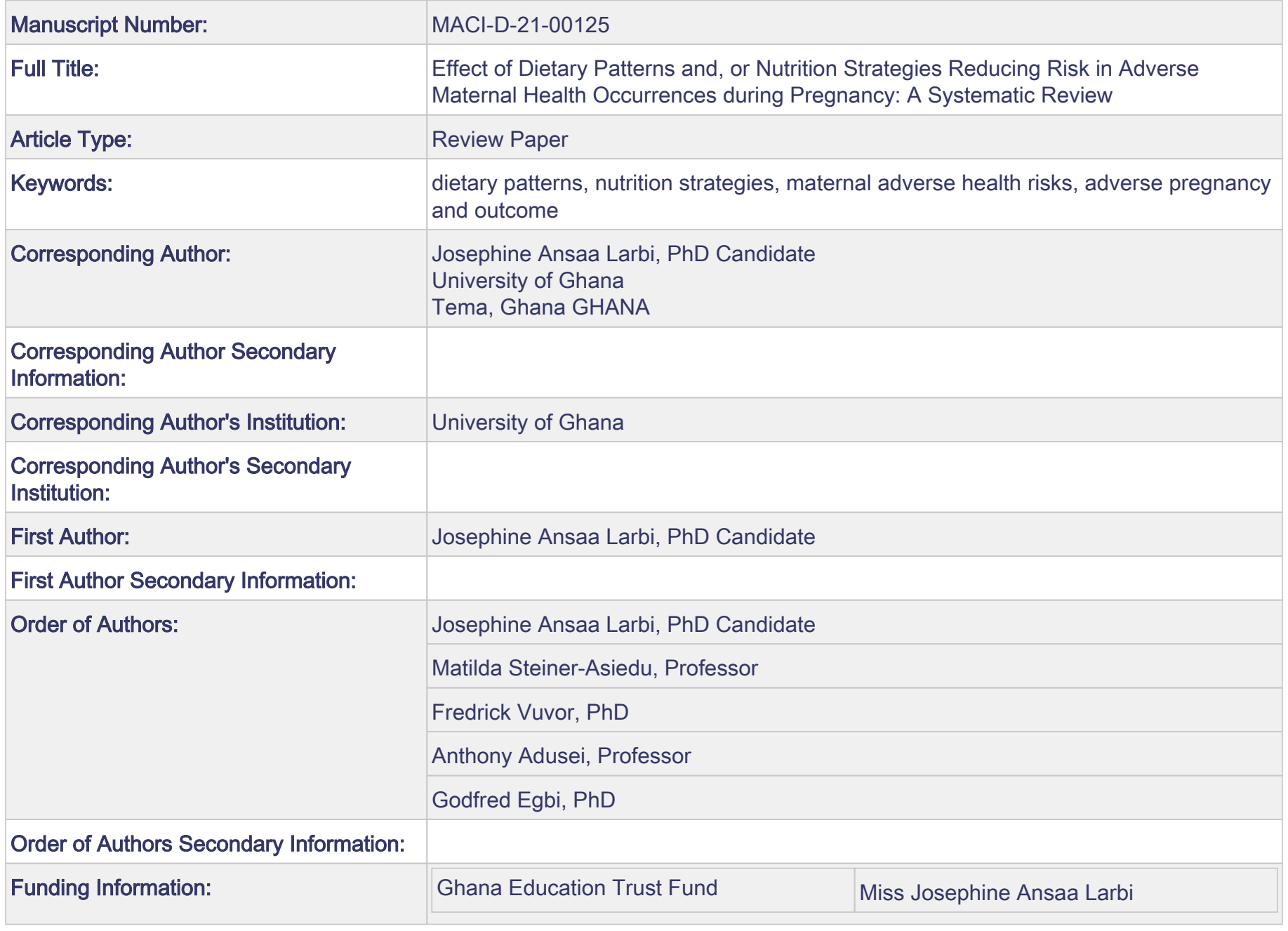




\section{Submission of a Manuscript to MCHJ}

Manuscript Title: Effect of Dietary Patterns and, or Nutrition Strategies Reducing Risk in Adverse Maternal Health Occurrences during Pregnancy: A Systematic Review

Author's Name: Josephine Ansaa Larbi (Ms)

$\mathrm{PhD}$ Candidate

Co-Authors Names: Matilda Steiner-Asiedu, Professor

Fredrick Vuvor, $\mathrm{PhD}$

Affiliation: University of Ghana, Accra

College of Basic and Applied Science

Department of Nutrition and Food Science

Co-Author: Anthony Kwaku Adusei, Professor

Affiliation: Kwame Nkrumah University of Science and Technology

School of Medical Science

Public Health

Co-Author: Godfred Egbi

Affiliation: Noguchi Memorial Institute

Nutrition Department

Ghana, Accra

E-mail: anyomijosephine23@gmail.com

Telephone number: +233-208177986 


\title{
Effect of Dietary Patterns and, or Nutrition Strategies Reducing Risk in Adverse Maternal Health Occurrences during Pregnancy: A Systematic Review
}

\begin{abstract}
Dietary patterns and nutrition strategies are essential to maintain optimal maternal health during pregnancy to prevent and minimize conditions that lead to complications. Previous studies found nutrition education and counseling effect on food habit modification, beneficial during pregnancy. In promoting health and reducing adverse maternal conditions during pregnancy, need increasing knowledge on diversities of dietary pattern consumption, modify attitude and practice. The present review conducted on effect of dietary patterns and/or nutrition interventions on health risks such as hypertension disorders in pregnancy or gestational hypertension or high blood pressure related to obesity or overweight, low haemoglobin, undernutrition and anaemia. Eighteen eligible studies identified. Studies on dietary patterns: Mediterranean or DASH diets, DASH diet vs Western diet with dietary counseling, combined nutrition counseling, advice and healthy diet. Healthy diet vs Western, Traditional or high-energy-foo; 2 healthy diets rich in fruit and vegetables. Two healthy diets combined with dietary counseling, nutrition messages compared with high-energy-food. The remaining 5 studies were nutrition education and health promotion model, dietary counseling and knowledge, attitude and practice with healthy diet, nutrition education with or without diet plan, then guided counseling with planned behaviour model. The studies reported positive impact on reduction of maternal adverse conditions, improved anthropometric, haemoglobin, undernutrition or malnutrition status. Study designs were 5 randomized controlled interventions, 11 prospective or longitudinal cohort studies and 2 systematic review.
\end{abstract}


Keywords: dietary patterns, nutrition strategies, maternal adverse health risks, adverse pregnancy and outcome

\section{Effect of Dietary Patterns and, or Nutrition Strategies Reducing Risk in Adverse Maternal Health Occurrences during Pregnancy: A Systematic Review \\ Declarations}

I declare that I have not received any support for funding.

This systematic review had no conflict of interest.

Articles reviewed sought ethical approval (include appropriate approvals or waivers). Not Applicable

Consent to participate (include appropriate consent statements). Not Applicable

Consent for publication (consent statement regarding publishing an individual's data or image). Not Applicable

Availability of data and material (data transparency) has been included in a table form in this reviewed article.

ORCID Code obtained after registration for a new author's manuscript for submission.

Authors' contributions not applicable. 


\section{Effect of Dietary Patterns and, or Nutrition Strategies Reducing Risk in Adverse Maternal Health Occurrences during Pregnancy: A Systematic Review}

\section{Significance:}

Nutrition plays a major role in maternal and child health. Healthy dietary pattern with adequate nutrition education and counseling reduce health risks of mothers and their unborn babies and maintain the well-being. Adverse health risks such as gestational hypertension or pregnancyinduce hypertension (PIH) or high blood pressure, undernutrition, malnutrition, low haemoglobin or anaemia, obesity and overweight in pregnancy are global health issue that need attention. This may be due to poor quality diet and could lead to complications and death if adequate attention is not giving during antenatal care.

Creating awareness for nutrition education, counseling and dietary patterns with reproductive women is health importance. Significantly, nutrition education and counseling improve maternal health in pregnancy by promoting, protecting, maintaining health and well-being and preventing health risks. Conducting a systematic review on effect of nutrition strategies and, or dietary patterns will show evidence of reducing or preventing maternal adverse health. This could help health professionals in developing countries to adopt strategies during antenatal management to improve maternal health status.

E-mail: anyomijosephine23@gmail.com 


\title{
Effect of Dietary Patterns and, or Nutrition Strategies Reducing Risk in Adverse Maternal Health Occurrences during Pregnancy: A Systematic Review
}

\begin{abstract}
Dietary patterns and nutrition strategies are essential to maintain optimal maternal health during pregnancy to prevent and minimize conditions that lead to complications. Previous studies found nutrition education and counseling effect on food habit modification, beneficial during pregnancy. In promoting health and reducing adverse maternal conditions during pregnancy, need increasing knowledge on diversities of dietary pattern consumption, modify attitude and practice. The present review conducted on effect of dietary patterns and/or nutrition interventions on health risks such as hypertension disorders in pregnancy or gestational hypertension or high blood pressure related to obesity or overweight, low haemoglobin, undernutrition and anaemia. Eighteen eligible studies identified. Studies on dietary patterns: Mediterranean or DASH diets, DASH diet vs Western diet with dietary counseling, combined nutrition counseling, advice and healthy diet. Healthy diet vs Western, Traditional or high-energy-foo; 2 healthy diets rich in fruit and vegetables. Two healthy diets combined with dietary counseling, nutrition messages compared with high-energy-food. The remaining 5 studies were nutrition education and health promotion model, dietary counseling and knowledge, attitude and practice with healthy diet, nutrition education with or without diet plan, then guided counseling with planned behaviour model. The studies reported positive impact on reduction of maternal adverse conditions, improved anthropometric, haemoglobin, undernutrition or malnutrition status. Study designs were 5 randomized controlled interventions, 11 prospective or longitudinal cohort studies and 2 systematic review.
\end{abstract}




\section{Effect of Dietary Patterns and, or Nutrition Strategies Reducing Risk in Adverse Maternal Health Occurrences during Pregnancy: A Systematic Review}

\section{Introduction}

It is evident that nutrition education, counseling and advice with nutritional support promote healthy state during pregnancy. ${ }^{1,2}$ Poor diet intakes and lifestyle affect physiological functions causing hypertension, malnutrition, undernutrition, anaemia, high body mass index, overweight and obesity in pregnancy. Severe maternal conditions can lead to premature delivery, low birth weight, maternal -foetal deaths. ${ }^{3,4}$ Aside inadequate nutrient intake affecting health, the biological utilization, physiological and pathological status of the individual is considered. Pregnant women consuming inadequate nutritious diet could develop health issues. The health problems resulting in pregnancy may attributing to inaccessibility, unaffordability, unacceptability and seasonal changes of food items leading to insecurity. Evidences are that diversities of dietary patterns and nutrition strategies reduce adverse maternal health conditions and prevent complications. ${ }^{5,6,7}$

In order to address most public health issues, behavioural counseling on dietary practices and nutrition education for females in reproductive age becomes necessary. Creating awareness on maternal-fetal risks relating to pre-pregnancy overweight that is potential for excessive gestational weight gain must need effective implementation to change individual's lifestyle. ${ }^{35}$ There is a need to focusing on individual's knowledge level, attitude and practices through motivation to achieve better food choice. Globally, gestational hypertension and pre-eclampsia represent 5-15\% and 8$10 \%$ respectively, which is due to hormonal changes causing increase blood pressure that lead to maternal mortality. ${ }^{8} 6$ Mostly, hypertension during pregnancy is diagnosed as blood pressure above 140/90mmHg after 20 weeks gestation without protein in urine and oedema. ${ }^{9,6}$ 
In Sub-Saharan Africa, pregnant women suffer from deficiencies of folate and iron because of inadequate nutritious diet leading to anaemia in pregnancy, morbidity, mortality and infections. ${ }^{10}$ World Health Organization (WHO) recommends nutrition education and counseling (NEC) adoption to increase healthy diets with fruit and vegetables to reduce health risks. (WHO, 2018). Fruit and vegetables contain minerals and vitamins that aid to reduce adverse conditions during pregnancy and birth outcomes. ${ }^{11,12}$ In order to upgrade knowledge of the inhabitants' in Iran, focusing on nutrition education campaign enable improvement in $\mathrm{F} \& \mathrm{~V}$ consumption to reduce nutrition-related-problems. ${ }^{13}$

World Health Organization and other studies improving knowledge on diet planning on food diversities, create awareness on sources of iron, folate, plant and animal food to reduce anaemia during pregnancy. ${ }^{14,15}$ It is evident, pregnant women with excessive vomiting or bleeding after 24 weeks gestation, may develop undernutrition, weight loss and anaemia. These conditions could cause premature delivery and low birth weight but intake of diversities of nutritious diet could improve their haemoglobin above $10.5 \mathrm{~g} / \mathrm{dl}$ and gain weight. ${ }^{16}$ Moreover, high-energy diets intake among African women in urban areas, contribute to obesity and overweight before becoming pregnant. This cause excessive weight gain during $1^{\text {st }}$ trimester. Women need encouragement to take diets rich in $\mathrm{F} \& \mathrm{~V}$ to reduce weight and complications in pregnancy ${ }^{17} \mathrm{WHO}$ recommends that health caregivers in clinics and communities implement effective nutrition advice, education and counseling in the antenatal management. ${ }^{1}$ Implementation of NEC in developing countries during antenatal care is inadequate or irregular. Therefore, reinforcing NEC and dietary interventions effectively could reduce health risks during pregnancy. ${ }^{18,19}$ Committing to dietary pattern and nutrition interventions as measures to reduce or prevent health risks in pregnancies need further investigation. 


\section{Effect of Dietary Patterns and, or Nutrition Strategies Reducing Risk in Adverse Maternal Health Occurrences during Pregnancy: A Systematic Review}

\section{Literature Search Method}

Literature searched from PubMed, Google Scholar and other similar reviewed studies conducted on dietary patterns, nutrition strategies and health risks during pregnancy. Selected study designs were randomized control trails (RCTs), cohort observational or prospective, quantitative and qualitative variables. Effect of randomized control trials on dietary approaches took minimum of 3 months, maximum less than one year and cohort observation took less than one year or more. Searched on dietary patterns interventions of DASH or Mediterranean, healthy diet with F\&V and related factors of overweight, high body mass index, hypertension, anaemia, undernutrition and malnutrition selected. Nutrition education and counseling interventions promoting healthy diet to reduce health problems were included. The search was restricted to articles published in English language. Titles, abstracts and full text relevant to the study reviewed with other cross-references screened for further search.

\section{Eligibility Criteria}

This was based on dietary patterns and, or nutrition strategy reducing health risk during pregnancy with birth outcomes. Studies included randomize control trial, quasi-experiment and prospective, longitudinal or observational cohort. Target population included pregnant women at 16 years or above aged 18, gestational age below 20 weeks and above, with problems such as hypertension, anaemia, obesity or overweight, low haemoglobin, undernutrition and malnutrition. High blood pressure defined above 140/90 $\mathrm{mmHg}$. Obesity and overweight definition according to WHO is measurement from $40 \mathrm{~kg} / \mathrm{m}^{2}$. Dietary patterns captured healthy diet, Mediterranean, DASH and traditional diet with fruit and vegetables. 


\section{Effect of Dietary Patterns and, or Nutrition Strategies Reducing Risk in Adverse Maternal Health Occurrences during Pregnancy: A Systematic Review}

Study interventions included healthy diets vs western foods, nutrition education and counseling, nutrition education or dietary counseling, diet planned and behaviour change model. Exclusion were non-pregnant women, pregnant teenagers, chronic diseases, non-intervention or intervention studies of dietary supplements or placebo (e.g. vitamin C, vitamin E, calcium, potassium, garlic, soy protein), or energy foods less than $600 \mathrm{kcal} /$ day, exercise as co-intervention, study designs of case control, retrospective cohort and clinical trials.

\section{Evaluation of Studies Results}

Reported study outcomes based on the following distinctions: i) difference between intervention and control groups with change from baseline to endline; ii) difference within groups from baseline to follow-up. iii) Differences between groups that considered change in values from baseline and endline adjusting cofounding characteristics to give meaningful comparison results. Differences considered as significant if the P-value less than 0.05 .

\section{Summary of Findings}

Relevant studies include titles, abstracts and full text extracted by first author's name, publication year, country, study designs, study length, participants' age, gestational age and sample size. The search criteria were dietary patterns, nutrition strategies, maternal health risks and pregnancy outcomes. Data extraction from PubMed, Google Scholar and related reviewed studies. Study designs involved 5 randomized controlled trials, 11 prospective or longitudinal cohort and 2 systematic reviews. Publications from 2006 to 2020 in USA, Australia, Boston, Canada, Denmark, Netherlands, India, Norway, Finland, Ethiopia and Cairo. Participants were pregnant women aged 16 years and above. Sample size between 100 to over 55,000, from a period of 3 days, 3, 6 and 


\section{Effect of Dietary Patterns and, or Nutrition Strategies Reducing Risk in Adverse Maternal Health Occurrences during Pregnancy: A Systematic Review}

and 24 months. Literature searched 25 articles, excluded 7 for undetailed information on titles and abstract or chronic conditions and rejected 4 duplicates of dietary patterns on hypertension with diabetes during pregnancy or food supplements. The relevant articles were 18, comprised of dietary patterns or nutrition strategies. For an overview of the screening criteria, see Figure 1.

\section{Data Extraction and Selection Process}

\begin{tabular}{|c|c|}
\hline Searched Articles=25 & \\
\hline $\begin{array}{l}\text { Eighteen eligible Articles Screened from Goggle and } \\
\text { PubMed: } \\
\text { Full text }=12, \text { Abstracts }=4 \text {, Systematic reviewed articles }=2\end{array}$ & $\begin{array}{c}\text { Reiected Articles: } \\
\text { Non eligibility Google }=4 ; \text { PubMed }=3\end{array}$ \\
\hline $\begin{array}{l}\text { Inclusion criteria: } \\
\text { Pregnant women with gestational hypertension/high blood } \\
\text { pressure, increased BMI/overweight, anaemia, underweight } \\
\text { or malnourished put on dietary patterns, nutrition education } \\
\text { or counseling. } \\
\text { Dietary patterns: Mediterranean only=2; DASH diet } \\
\text { only=1, Healthy diet only=1, Mediterranean vs Traditional } \\
\text { diet=2, dietary counseling + DASH vs Western diet or } \\
\text { Healthy diet vs high-energy food=2, Nutrition Counseling } \\
\text { +advice +healthy diet=1, Sea food +fish +vegetables=1, } \\
\text { Healthy diet vs Western/Tradition/high-energy food=2; } \\
\text { Nutrition strategies + healthy diets + KAP=2, Nutrition } \\
\text { strategies only=4. } \\
\text { Study design: randomize control trial, prospective cohort; } \\
\text { observational/longitudinal }\end{array}$ & $\begin{array}{l}\text { Exclusion: conditions with chronic } \\
\text { hypertension, eclampsia }=3 \\
\text { Rejected duplicates: dietary pattern with } \\
\text { hypertension-diabetes during pregnancy or } \\
\text { food supplements }=4\end{array}$ \\
\hline
\end{tabular}

Figure 1 Flow chart showing screening process for eligible and non-eligible articles. 


\section{Effect of Dietary Patterns and, or Nutrition Strategies Reducing Risk in Adverse Maternal Health Occurrences during Pregnancy: A Systematic Review}

Eligible studies investigated were 18. All the studies measured significant improvement in specific health problem in intervention and control groups. The review investigated a decreased in health risks with 9 dietary patterns, 5 nutrition strategies with dietary pattern and 4 nutrition strategies promoted healthy diet. Studies conducted in 4 developing countries, two systematics and 12 from developed nations, communities and health facilities showed specific effect of dietary patterns or nutrition strategies.

\section{Relevant Discussions}

\section{Effect of Dietary Patterns on Maternal Health Problems}

Three of seven studies investigated effect of high Mediterranean, DASH, healthy diet and seafoodvegetables, found odds risk below 0.8 reduced hypertension in pregnancy. ${ }^{20,21,22}$ Out of the remaining studies, a healthy diet reduced hypertension disorders in pregnancy (HDP) by odds 0.72 compared with energy food, which increased odds by $1.21 .^{23}$

A high or low Mediterranean diet respectively lowered risk by $47 \%$ of HDP and increased prepregnancy BMI by $22 \%$, while western, traditional or high-energy food increased risk of high blood pressure at 20 and 30 weeks gestation. ${ }^{24,25,26}$

Different nutrition interventions showed positive effect on reduction in undernutrition, anaemia, excessive weight gain and high blood pressure. ${ }^{27,15,32,28}$ It aid decreased intake of energy-foods to reduce obesity or high body mass index. ${ }^{7}$ It also improve food portions, haemoglobin level and normal weight gain during pregnancy. ${ }^{29,30,31,32}$ 


\section{Effect of Dietary Patterns and, or Nutrition Strategies Reducing Risk in Adverse Maternal Health Occurrences during Pregnancy: A Systematic Review \\ Effect of Dietary Patterns and Counseling on Anthropometric Status}

Interventional effect of nutrition education or dietary counseling on dietary practices improved weight and reduced excessive weight or BMI. One study increased knowledge on daily food quantity. ${ }^{29}$ The remaining respectively reduced excessive gestational weight gain by $42.7 \%$ and $13.9 \%$, with mean weight reduction of $51.05 \pm 7.26 \mathrm{~kg}$ while another improved haemoglobin and reduced anaemia in intervention vs control group. ${ }^{15,30,32}$

A study found that DASH diet intake was ineffective in early pregnancy against HDP, but had a decreased effect on obesity before pregnancy and subsequent gestational weight gain. ${ }^{28}$ A metaanalysis on effective combination of NEC with nutritional support found an improved weight gain during pregnancy. ${ }^{31}$

\section{Effect of Nutritional Education-Counseling on Dietary Intake and Practices}

Three studies conducted on dietary counseling and nutrition education among pregnant women during antenatal visits, enabled upgrading of knowledge on nutrition health benefits and modified dietary practices. ${ }^{7,15,30}$ One study showed impact of nutrition education with health promotion model to improving dietary practices on intake of food diversities among pregnant women. Systematic reviewed studies conducted on nutrition education messages and counseling found promoting effective dietary practices to improve weight gain and reduced risk of anaemia. ${ }^{29,31}$

\section{Effect on Haemoglobin Level, Anaemia and Undernutrition}

A study reported on significant beneficial effect of nutrition education messages and counseling on food quality, quantity and undernutrition. This showed mean body weight of $51.05 \pm 7.26 \mathrm{~kg}$, haemoglobin status at $9.65 \pm 0.97$ and $7.85 \pm 1.58$ with reduction in anaemia prevalence from 


\section{Effect of Dietary Patterns and, or Nutrition Strategies Reducing Risk in Adverse Maternal Health Occurrences during Pregnancy: A Systematic Review}

$96.3 \%$ to $78.7 \%$ and $96.0 \%$ at post nutrition education and non-nutrition education, p-value $<0.001 .^{32}$

In addition, a nutrition education on source of iron food and diet planned respectively found significant mean haemoglobin level of $0.56 \pm 0.40 \mathrm{gm} / \mathrm{dl}$ and $0.16 \pm 0.82 / \mathrm{dl}$ in intervention and control groups. ${ }^{15}$ The study also improved knowledge by $8.26 \pm 4.57$ and $1.05 \pm 6.59$ respectively and anaemia from $40 \mathrm{gm} / \mathrm{dl}$ to $82 \mathrm{gm} / \mathrm{dl}$, p-value $<0.002 .^{15}$

Another study reported on undernutrition at base-line prevalence of $47.3 \%$ and adopting guided counseling with theory of planned behaviour reduced to $30.6 \%$ at endline. ${ }^{27}$ This also showed a changed of $16.7 \%$ in intervention than $43.8 \%$ to $39.8 \%$ in control group. ${ }^{27}$

\section{Effect on Birth Outcome}

Two studies reported on birth outcomes. A nutrition education with nutritional support upgraded knowledge on diet that decreased anaemia, increased birth weight to $105 \mathrm{~g}$ and reduced $19 \%$ of premature birth than applied NEC only. ${ }^{31}$ On the other hand, DASH diet intake showed 0.59 odds risk reduction in premature birth in intervention than 1.55 in control group. ${ }^{33}$ 


\section{Effect of Dietary Patterns and, or Nutrition Strategies Reducing Risk in Adverse Maternal Health Occurrences during Pregnancy: A Systematic Review}

\section{Summary:}

\begin{tabular}{|c|c|c|c|c|c|}
\hline References & $\begin{array}{l}\text { Study } \\
\text { design, } \\
\text { Age,Sampl } \\
\text { e size }\end{array}$ & $\begin{array}{l}\text { Nutrition } \\
\text { Strategies }\end{array}$ & $\begin{array}{l}\text { Dietary } \\
\text { Patterns }\end{array}$ & $\begin{array}{l}\text { Method/ } \\
\text { Duration }\end{array}$ & Results /Information \\
\hline $\begin{array}{l}\text { 1. Jarman et } \\
\text { al, } 2018 \\
\text { Alberta, } \\
\text { Canada; }\end{array}$ & $\begin{array}{l}\begin{array}{l}\text { Prospective } \\
\text { cohort, }\end{array} \\
1545 \\
\text { pregnant } \\
\text { women, } \\
\text { Above } 16 \\
\text { years, } \\
17 \text { - } \\
27 \text { weeks } \\
\text { gestation. }\end{array}$ & None & $\begin{array}{l}\text { 1. healthy diet } \\
\text { 2. Meat, refined } \\
\text { carbohydrate, } \\
\text { 3. Beans, cheese } \\
\text { 4.Salad/tea/ } \\
\text { coffee }\end{array}$ & $\begin{array}{l}\text { Food } \\
\text { frequency } \\
\text { questionna } \\
\text { ire (FFQ), } \\
12 \text { months }\end{array}$ & $\begin{array}{l}\text { High healthy diet intake lowered } \\
\text { gestational hypertension }(\mathrm{GH}) \\
93(6 \%) \text { by odds } 0.6\end{array}$ \\
\hline $\begin{array}{l}\text { 2. Ikem, et al., } \\
2019 . \\
\text { Denmark }\end{array}$ & $\begin{array}{l}\text { Prospective } \\
\text { longitudina } \\
1 \text { Cohort } \\
55,138 \\
\text { women } 12 \\
\& 30 \text { weeks } \\
\text { pregnant }\end{array}$ & None & $\begin{array}{l}\text { 1. Western diet, } \\
\text { 2.Seafood, } \\
\text { vegetables, fish }\end{array}$ & $\begin{array}{l}\text { Interview- } \\
\text { questionna } \\
\text { ire, } \\
6 \& 18 \\
\text { months }\end{array}$ & $\begin{array}{l}\text { Seafood/vegetables } \\
\& \text { fish decreased GH by } 0.86 \text { odds. } \\
\text { Western-diet increased GH by } \\
1.18 \text { odds }\end{array}$ \\
\hline $\begin{array}{l}\text { 3. Girard \& } \\
\text { Olude, } 2012\end{array}$ & $\begin{array}{l}\text { Systematic } \\
\text { review } \\
3877 \\
\text { publication } \\
\text { s }\end{array}$ & $\begin{array}{l}\text { Nutrition } \\
\text { education } \\
\& \\
\text { counsellin } \\
\text { g (NEC) }\end{array}$ & $\begin{array}{l}\text { Dietary practices } \\
+ \text { iron- folic acid } \\
\text { (IFA) } \\
\text { supplementation }\end{array}$ & $2010-2011$ & $\begin{array}{l}\text { Health-education messages, NEC } \\
\& \text { nutrition support improved } \\
\text { weight-gain } \\
(0.45 \mathrm{~kg} \text {,intervention }), \\
(0.15 \mathrm{~kg} \text {,control }) \text { groups, } \\
30 \% \text { reduction risk in anaemia. }\end{array}$ \\
\hline $\begin{array}{l}\text { 4. Garg, et al., } \\
\text { 2006. India }\end{array}$ & $\begin{array}{l}\text { Randomize } \\
\text { control trial } \\
100 \\
\text { pregnant } \\
\text { women }\end{array}$ & $\begin{array}{l}\text { Nutrition } \\
\text { education }\end{array}$ & None & $\begin{array}{l}\text { Nutrition } \\
\text { messages } \\
10- \\
16 \text { weeks } \\
\text { 24-hour } \\
\text { recall, } \\
\text { FFQ }\end{array}$ & $\begin{array}{l}\text { Mean body weight } \\
=(51.05 \pm 7.26 \mathrm{~kg}) \text {. } \\
\text { Mean haemoglobin increased } \\
\text { Post-NE }=9.65 \pm 0.97 \\
\text { Non-NE }=7.85 \pm 1.58, \mathrm{p}<0.001) \\
\text { Anaemia prevalence reduction, } \\
\mathrm{PNE}=96 \% \\
\mathrm{NNE}=78.7 \%\end{array}$ \\
\hline $\begin{array}{l}\text { 5.Piirainen, et } \\
\text { al,2006. }\end{array}$ & $\begin{array}{l}\text { Prospective } \\
\text { cohort } \\
\text { study. } 209\end{array}$ & $\begin{array}{l}\text { Dietary } \\
\text { counseling }\end{array}$ & $\begin{array}{l}\text { Randomized } \\
\text { intervention } \\
\text { (healthy diet) }\end{array}$ & $\begin{array}{l}\text { Three-day } \\
\text { food } \\
\text { records }\end{array}$ & $\begin{array}{l}\text { A difference of } 0.5 \% \text { intake of } \\
\text { PUFA and } 0.8 \% \text { reduction intake } \\
\text { of SFA in intervention group }\end{array}$ \\
\hline
\end{tabular}




\begin{tabular}{|c|c|c|c|c|c|c|c|}
\hline $\begin{array}{l}\text { Britain- } \\
\text { Findland }\end{array}$ & $\begin{array}{l}\text { pregnant } \\
\text { women. }\end{array}$ & $\begin{array}{l}\text {,practices } \\
\text { \& advice }\end{array}$ & $\begin{array}{l}\text { Control(dietary } \\
\text { habit/food-diary) }\end{array}$ & $\begin{array}{l}\text { 12weeks } \\
\text { interventio } \\
\mathrm{n}\end{array}$ & & & \\
\hline $\begin{array}{l}\text { 6. Fulay, et al, } \\
\text { 2018. Boston }\end{array}$ & $\begin{array}{l}\text { longitudina } \\
1 \text { cohort } \\
-1760 \\
\text { pregnant } \\
\text { women, } \\
\text { Mean SD } \\
\text { age } \\
32.2 \pm 4.9 \\
\text { years }\end{array}$ & None & DASH diet & $\begin{array}{l}\text { FFQ } \\
-11.1 \\
\text { weeks } \\
\text { gestation. }\end{array}$ & \multicolumn{3}{|c|}{$\begin{array}{l}\text { Mean DASH score: } 24.0, \text { SD: } 5.0 \\
\text { not associated with pregnancy } \\
\text { outcomes } \\
-0.19 \mathrm{~kg} \text { weight reduction. } \\
\mathrm{p} \leq 0.05\end{array}$} \\
\hline \multirow{7}{*}{$\begin{array}{l}\text { 7. Goodarzi- } \\
\text { Khoigani, et } \\
\text { al., 2018. Iran }\end{array}$} & \multirow{7}{*}{$\begin{array}{l}\text { Randomize } \\
\text { d Clinical } \\
\text { Trial } \\
\text { Pregnant } \\
\text { women: 6- } \\
10 \text { weeks }\end{array}$} & \multirow{7}{*}{$\begin{array}{l}\text { Nutrition } \\
\text { education } \\
\& \\
\text { nutrition } \\
\text { plan }\end{array}$} & \multirow[t]{7}{*}{$\begin{array}{l}\text { 3-days dietary } \\
\text { record }\end{array}$} & \multirow{7}{*}{$\begin{array}{l}16 \text { weeks } \\
\text { interventio } \\
\mathrm{n} \\
\text { Gestation } \\
6-10 \\
\text { weeks \& } \\
34-36 \\
\text { weeks. }\end{array}$} & \multicolumn{3}{|c|}{$\begin{array}{l}\text { Mean-standard deviation (SD) of } \\
\text { food portions }\end{array}$} \\
\hline & & & & & Food & $\begin{array}{l}\text { Interventi } \\
\text { on } \\
\text { improved }\end{array}$ & Control \\
\hline & & & & & grain & $10.40(1.96)$ & $12.70(1.93)$ \\
\hline & & & & & $\begin{array}{l}\text { vegeta } \\
\text {-ble }\end{array}$ & $3.88(1.33)$ & $2.96(0.91)$ \\
\hline & & & & & fruit & $4.02(0.05)$ & $3.95(0.91)$ \\
\hline & & & & & dairy & $2.33(0.68)$ & $2.11(0.45)$ \\
\hline & & & & & meat & $3.17(0.68)$ & $2.96(0.67)$ \\
\hline \multirow{5}{*}{$\begin{array}{l}\text { 8. Martin, et } \\
\text { al., } 2015 . \\
\text { US-North } \\
\text { Carolina }\end{array}$} & \multirow{5}{*}{$\begin{array}{l}\text { Prospective } \\
\text { cohort } \\
-3143 \\
\text { pregnant } \\
\text { women. } \\
\text { 19-44 yrs } \\
26-29 \text { wks }\end{array}$} & \multirow{5}{*}{$\begin{array}{l}\text { Dietary } \\
\text { counseling } \\
\text {-dietary } \\
\text { patterns }\end{array}$} & \multirow[t]{5}{*}{ DASH diet } & \multirow{5}{*}{$\begin{array}{l}\text { Interviews } \\
\text { and self- } \\
\text { administer } \\
\text { ed } \\
\text { questionna } \\
\text { ires FFQ } \\
1995-2000 \\
2001-2005\end{array}$} & \multicolumn{3}{|c|}{ Mean DASH scores $=24.3 \pm 4.9$} \\
\hline & & & & & \begin{tabular}{|l|} 
Odds \\
Ratio
\end{tabular} & \begin{tabular}{|l|} 
High \\
DASH
\end{tabular} & $\begin{array}{l}\text { Low } \\
\text { DASH }\end{array}$ \\
\hline & & & & & 0.59 & $\begin{array}{l}\text { Low } \\
\text { preterm }\end{array}$ & - \\
\hline & & & & & 1.55 & - & $\begin{array}{l}\text { High } \\
\text { preterm }\end{array}$ \\
\hline & & & & & & & \\
\hline $\begin{array}{l}\text { 9. Ramkripa, } \\
\text { et al., } 2019\end{array}$ & $\begin{array}{l}\text { A } \\
\text { systematic } \\
\text { review } \\
9103 \\
\text { studies } \\
\text { Cohort and } \\
\text { randomize } \\
\text { d control } \\
\text { trial } \\
\text {-pregnant } \\
\text { women }\end{array}$ & None & $\begin{array}{l}\text { Healthy dietary } \\
\text { patterns }\end{array}$ & $\begin{array}{ll}1980- \\
2017\end{array}$ & \multicolumn{3}{|c|}{ Lowered risk of HDP } \\
\hline $\begin{array}{l}10 . \\
\text { Schoenaker, et }\end{array}$ & $\begin{array}{l}\text { Observatio } \\
\text { nal- }\end{array}$ & None & $\begin{array}{l}\text { Mediterranean } \\
\text { diet }\end{array}$ & $\begin{array}{l}\text { FFQ, } \\
2003-2012\end{array}$ & \multicolumn{3}{|c|}{$\begin{array}{l}\text { High Mediterranean } \\
\text { diet reduced risk of developing } \\
\text { HDPs in } 305(8.5 \%) \text { pregnancies }\end{array}$} \\
\hline
\end{tabular}




\begin{tabular}{|c|c|c|c|c|c|}
\hline $\begin{array}{l}\text { al., } 2015 . \\
\text { Australia }\end{array}$ & $\begin{array}{l}\text { Longitudin } \\
\text { al Study } \\
\text { 3582pregn } \\
\text { ant women }\end{array}$ & & & & -RR, 0.58; 95\% CI, 0.42, 0.81). \\
\hline $\begin{array}{l}11 . \\
\text { Schoenaker, et } \\
\text { al., } 2016 . \\
\text { Australia }\end{array}$ & $\begin{array}{l}\text { Longitudin } \\
\text { al Study } \\
3378 \\
\text { pregnant } \\
\text { women } \\
25-30 \text { years }\end{array}$ & None & $\begin{array}{l}\text { Mediterranean } \\
\text { dietary score }\end{array}$ & $\begin{array}{l}\text {-FFQ } \\
2003- \\
2012 .\end{array}$ & $\begin{array}{l}273(8.6 \%) \text { adhered to low } \\
\text { Mediterranean diet associated } \\
\text { with higher risk of HDP } \\
\text { OR: } 1.41,1.18,1.56 \text {, } \\
\text { BMI } 1-\mathrm{kg} / \mathrm{m}^{(2)} \text { increased by } 22 \% \text {. }\end{array}$ \\
\hline $\begin{array}{l}12 . \\
\text { Timmermans, } \\
\text { et al., 2011 } \\
\text { Dutch- } \\
\text { Netherlands }\end{array}$ & $\begin{array}{l}\text { Prospective } \\
\text { study } \\
-3187 \\
\text { pregnant } \\
\text { women }\end{array}$ & None & $\begin{array}{l}\text {-Low } \\
\text { Mediterranean } \\
\text {-Traditional diet. }\end{array}$ & $\begin{array}{l}\text { Semi- } \\
\text { quantitativ } \\
\text { e } \\
- \text { FFQ } \\
-3 \text { months }\end{array}$ & $\begin{array}{l}\text { High intake Traditional diet } \\
\text { increased SBP by } 1.8,2.3,2.6 \\
\text { mmHg } \\
\text {-low Mediterranean diet increased } \\
\text { SBP by } 1.1,2.2,1.3 \mathrm{mmHg}, \text { (early, } \\
\text { mid \& late pregnancies } \\
\text { - DBP decreased by } \\
1.3,1.6,2.4 \mathrm{mmHg} \text { respectively }\end{array}$ \\
\hline $\begin{array}{l}13 . \\
\text { Timmermans, } \\
\text { et al., } 2012 \\
\text { Dutch- } \\
\text { Netherlands }\end{array}$ & $\begin{array}{l}\text { Prospective } \\
\text { cohort } \\
\text { study }\end{array}$ & None & $\begin{array}{l}\text { High } \\
\text { Mediterranean } \\
- \\
\text { Western/Traditio } \\
\text { nal diets }\end{array}$ & $\begin{array}{l}\text { Semi- } \\
\text { quantitativ } \\
\text { e FFQ }\end{array}$ & $\begin{array}{l}\text { High Mediterranean diet } \\
\text { decreased } 12.8 \% \text { blood pressure } \\
\text { compared to } 8.7 \% \text { of high } \\
\text { Traditional diet }\end{array}$ \\
\hline $\begin{array}{l}14 . \\
\text { Timmermans } \\
\text { et al., } 2012 \text {. } \\
\text { Dutch- } \\
\text { Netherlands }\end{array}$ & $\begin{array}{l}\text { Prospective } \\
\text { cohort } \\
\text { study }\end{array}$ & None & $\begin{array}{l}\text { Healthy diet } \\
\text { Western-high } \\
\text { energy diet } \\
\text { Traditional diet, }\end{array}$ & $\begin{array}{l}\text { Semi- } \\
\text { quantitativ } \\
\text { e FFQ }\end{array}$ & $\begin{array}{l}\text { High healthy diet reduced GH } \\
\text {-Western or high-energy diet or } \\
\text { Traditional diet increased risk of } \\
\text { developing GH. }\end{array}$ \\
\hline $\begin{array}{l}\text { 15.Brantsaeter } \\
\text { et al., } 2009 . \\
\text { Norway }\end{array}$ & $\begin{array}{l}\text { Cohort } \\
\text { Study, } \\
23,423 \\
\text { nulliparous } \\
15,17-22 \\
\text { gestations }\end{array}$ & None & $\begin{array}{l}\text { - High Plant food } \\
\text { - High energy- } \\
\text { food }\end{array}$ & $\begin{array}{l}\text {-FFQ } \\
2007-2008\end{array}$ & $\begin{array}{l}\text { Plant food decreased risk (OR } \\
0.72,0.62 ; 0.85) \text {, } \\
\text { Energy-food increased risk (OR } \\
1.21 ; 1.03,1.42) \text {, }\end{array}$ \\
\hline $\begin{array}{l}\text { 16. Abdel- } \\
\text { Aziz, et al., } \\
\text { 201. Cairo }\end{array}$ & $\begin{array}{l}\text { Randomize } \\
\text { study } \\
200 \\
\text { primigravi } \\
\text { dae } \\
20-30 \text { years }\end{array}$ & $\begin{array}{l}\text { Dietary/ } \\
\text { Nutrition } \\
\text { counseling }\end{array}$ & -Healthy diet & $\begin{array}{l}\text {-Follow } \\
\text { up at ANC } \\
2015- \\
2016\end{array}$ & $\begin{array}{l}\text { Gestational weight gain } \\
42.7 \%=\text { intervention } \\
13.7 \%=\text { control group } \\
\text { - improved healthy diet intake, } \\
\text { Knowledge, Attitude, Practice } \\
-P \text {-value }<0.001 .\end{array}$ \\
\hline $\begin{array}{l}\text { 17. Sunuwar, } \\
\text { et al., } 2019 . \\
\text { Nepal }\end{array}$ & $\begin{array}{l}\text {-Quasi- } \\
\text { experiment } \\
\text {-Pregnant } \\
\text { women } \\
\text { aged 15-49 }\end{array}$ & $\begin{array}{l}\text {-Nutrition } \\
\text { education } \\
\text {-diet plan }\end{array}$ & $\begin{array}{l}\text { Semi-structured } \\
\text { questionnaire }\end{array}$ & $\begin{array}{l}\text {-Follow- } \\
\text { up every } 4 \\
\text { weeks for } \\
10 \text { weeks } \\
2017- \\
2018\end{array}$ & $\begin{array}{l}\text { Nutritional knowledge } \\
\text { anaemia \& iron-rich-foods, } \\
\text { showed } 66.0 \% \text { (intervention) } \\
24.1 \% \text { (control group) } \\
=40 \mathrm{gm} / \mathrm{dl} \text { to } 82 \mathrm{gm} / \mathrm{dl} . \\
\text { P-value }=0.002\end{array}$ \\
\hline
\end{tabular}




\begin{tabular}{|c|c|c|c|c|c|}
\hline & $\begin{array}{l}\text { mild- } \\
\text { moderate } \\
\text { anaemia } \\
\end{array}$ & & & & \\
\hline $\begin{array}{l}\text { 18. Demilew } \\
\text { et al., } 2020 . \\
\text { West Gojjam } \\
\text { Ethiopia }\end{array}$ & $\begin{array}{l}\text { Cluster- } \\
\text { randomize } \\
\text { control } \\
-694 \\
\text { pregnant } \\
\text { women(6w } \\
\text { ks) }\end{array}$ & $\begin{array}{l}\text { Guided } \\
\text { counseling }\end{array}$ & $\begin{array}{l}\text { Structured } \\
\text { questionnaire }\end{array}$ & $\begin{array}{l}4- \\
\text { counseling } \\
\text { sessions } \\
2018- \\
2019 .\end{array}$ & $\begin{array}{l}\text { Undernutrition prevalence }=16.7 \% \\
\text { reduction in intervention }\end{array}$ \\
\hline
\end{tabular}


Effect of Dietary Patterns and, or Nutrition Strategies Reducing Risk in Adverse Maternal Health Occurrences during Pregnancy: A Systematic Review

Assessment on Specific Effects

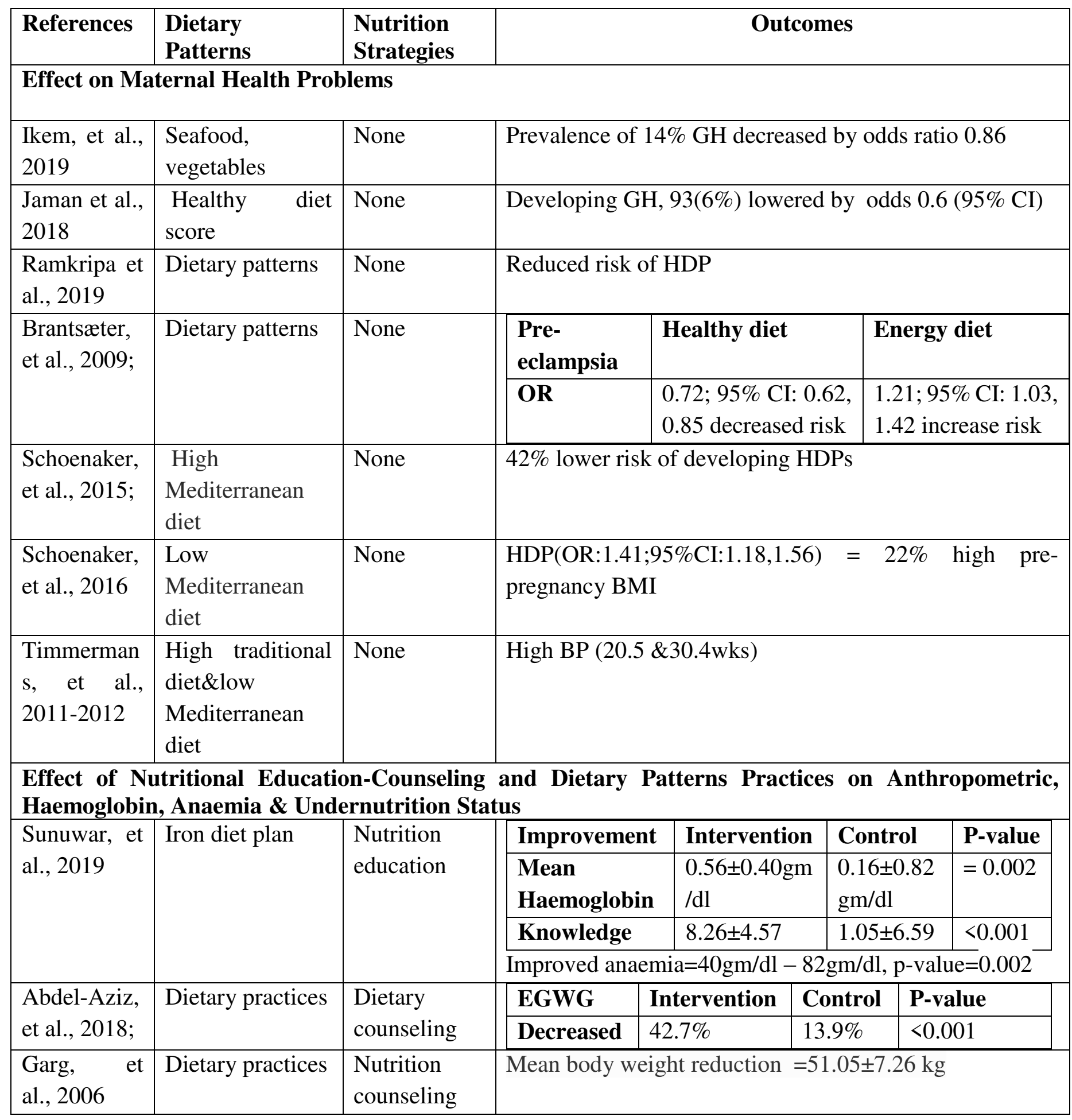




\begin{tabular}{|c|c|c|c|c|c|c|c|c|c|}
\hline $\begin{array}{l}\text { Fulay, et al., } \\
2018\end{array}$ & DASH diet score & None & \multicolumn{7}{|c|}{$\begin{array}{l}\text { Pregnancy complications } \neq \text { Mean DASH score }(24.0, \text { SD: } \\
5.0)=0.19(95 \% \text { CI: } 0.05,0.34), \mathrm{P} \leq 0.05 \mathrm{~kg} \text { higher GWG. }\end{array}$} \\
\hline $\begin{array}{l}\text { Piirainen, et } \\
\text { al., 2006; }\end{array}$ & Food products & $\begin{array}{l}\text { Dietary } \\
\text { counseling }\end{array}$ & \multicolumn{7}{|c|}{$\begin{array}{l}\text { Health benefits of } 0.5 \% \text { (PUFA) difference, } \\
0.8 \% \text { lower (SFA) in intervention group }\end{array}$} \\
\hline \multirow{6}{*}{$\begin{array}{l}\text { Goodarzi- } \\
\text { Khoigani, et } \\
\text { al., } 2018\end{array}$} & \multirow[t]{6}{*}{ Nutrition plan } & \multirow[t]{6}{*}{$\begin{array}{l}\text { Nutrition } \\
\text { education }\end{array}$} & \multicolumn{4}{|c|}{$\begin{array}{l}\text { Mean standard deviation } \\
\text { (SD) of food portions }\end{array}$} & \multicolumn{2}{|c|}{$\begin{array}{l}\text { Intervention } \\
\text { (improved) }\end{array}$} & Control \\
\hline & & & \multicolumn{4}{|l|}{ grain } & \multicolumn{2}{|c|}{$10.40(1.96)$} & $12.70(1.93)$ \\
\hline & & & \multicolumn{4}{|l|}{ vegetable } & \multicolumn{2}{|c|}{$3.88(1.33)$} & $2.96(0.91)$ \\
\hline & & & \multicolumn{4}{|l|}{ fruit } & \multicolumn{2}{|c|}{$4.02(0.05)$} & $3.95(0.91)$ \\
\hline & & & \multicolumn{4}{|l|}{ dairy } & \multicolumn{2}{|c|}{$2.33(0.68)$} & $2.11(0.45)$ \\
\hline & & & \multicolumn{4}{|l|}{ meat } & \multicolumn{2}{|c|}{$3.17(0.68)$} & $2.96(0.67)$ \\
\hline $\begin{array}{l}\text { Girard } \& \\
\text { Olude, } 2012\end{array}$ & Diversity foods & NEC & \multicolumn{3}{|c|}{$\begin{array}{l}\text { Improved } \\
\text { Weight gain } \\
\text { Anaemia }(30 \%)\end{array}$} & \multicolumn{4}{|c|}{\begin{tabular}{cc|} 
NEC (intervention) & (control) \\
$0.45 \mathrm{~kg}$ & $0.15 \mathrm{~kg}$
\end{tabular}} \\
\hline \multirow[t]{4}{*}{$\frac{\text { Demilew, et }}{\text { al. } 2020}$} & \multirow[t]{4}{*}{ None } & \multirow{4}{*}{$\begin{array}{l}\text { Guided } \\
\text { Counseling\& } \\
\text { Theory of } \\
\text { planned } \\
\text { behaviour }\end{array}$} & $\begin{array}{l}\text { Prevalence } \\
\text { of under- } \\
\text { nutrition }\end{array}$ & \multicolumn{3}{|c|}{ intervention } & \multicolumn{2}{|c|}{ control } & p-value \\
\hline & & & Baseline & \multicolumn{3}{|c|}{$47.3 \%$} & \multicolumn{2}{|c|}{$43.8 \%$} & 0.28 \\
\hline & & & Endline & \multicolumn{3}{|c|}{$30.6 \%$} & \multicolumn{2}{|c|}{$39.8 \%$} & $<0.00$ \\
\hline & & & Difference & \multicolumn{3}{|c|}{$\begin{array}{l}16.7 \% \\
\text { (reduction) }\end{array}$} & & & \\
\hline \multirow[t]{4}{*}{$\begin{array}{l}\text { Garg, et } \\
\text { al., } 2006\end{array}$} & \multirow[t]{4}{*}{ Dietary practices } & \multirow[t]{4}{*}{$\begin{array}{l}\text { Nutrition } \\
\text { counseling }\end{array}$} & \multicolumn{7}{|c|}{$\begin{array}{l}\text { Increased diet quality \& quantity \& } 96.3 \% \text { anaemia } \\
\text { prevalence reduced }\end{array}$} \\
\hline & & & \multicolumn{3}{|l|}{ Strategies } & Pos & $\mathbf{t} \mathrm{NE}$ & & Jon NE \\
\hline & & & Anaemia & & & & $.7 \%$ & & $96 \%$ \\
\hline & & & Mean hemog & lob & & 9.6 & \pm 0.97 & & $85 \pm 1.58$ \\
\hline Effect on Bir & h Outcomes & & & & & & & & \\
\hline $\begin{array}{l}\text { Girard \& } \\
\text { Olude, } 2012\end{array}$ & $\begin{array}{l}\text { Nutrition support } \\
\text { most significance }\end{array}$ & $\begin{array}{l}\mathrm{NEC}=\text { no } \\
\text { significance }\end{array}$ & $\begin{array}{l}\text { Birthweight ra } \\
\text { preterm delive }\end{array}$ & $\mathrm{r}$ & $\begin{array}{l}\mathrm{d}= \\
\mathrm{edu}\end{array}$ & $1-1$ & & & \\
\hline Martin, et & DASH diet & None & Odds risk & & $\mathrm{Hi}$ & adh & rence & Low a & dherence \\
\hline al., 2015 & & & Preterm bir & & $\begin{array}{l}0.5 \\
95\end{array}$ & & $0,0.85$ & $\begin{array}{l}1.55 \\
95 \% \mathrm{C}\end{array}$ & $\mathrm{I}: 1.07,2.24$ \\
\hline
\end{tabular}




\section{Effect of Dietary Patterns and, or Nutrition Strategies Reducing Risk in Adverse Maternal Health Occurrences during Pregnancy: A Systematic Review \\ Relevance and Limitations of Literature Review}

Reported nine studies on healthy dietary patterns improved maternal health status while other 9 studies on nutrition strategies modified food habit, improved knowledge and practices to maintain healthy lifestyle. One study found that DASH diet had no association with HDP, gestational diabetes, premature delivery or birth size but a positive effect on weight reduction related to obesity. ${ }^{28}$ Individual weekly reinforcement on dietary practices, nutrition education or counseling showed beneficial effect on health during pregnancy. Studies on dietary practices and nutrition strategies showed effective reduction in excessive gestational weight gain that could decreased poor birth outcomes. ${ }^{30,31,32}$ Dietary counseling and nutrition education served as preventive measures in medicine: protecting, promoting and maintaining healthy lifestyle and well-being. Therefore, implementation of dietary counseling with health promotion could modify understanding for healthy living and effective behavioural change during pregnancy. A Cochrane systematic review and meta-analysis supported that healthy dietary patterns decrease HDP, gestational diabetes mellitus, premature birth and low birth weight (Kibret, et al., 2018).This systematic reviewed showed significant effect of dietary patterns or nutrition strategies on maternal health problems with $\mathrm{P}$-value $<0.001$ to $<0.05$ which is a strong scientific evidence. Further investigation needed for how food portions intake could have impacts on pregnancy outcomes. Most of the studies conducted in developed countries related to well-educated people could not be generalized. Further research need investigation into situations in developing countries to understand effect of inadequate resources and food insecurity on dietary intake and adverse pregnancy or birth outcomes. 


\section{Effect of Dietary Patterns and, or Nutrition Strategies Reducing Risk in Adverse Maternal Health Occurrences during Pregnancy: A Systematic Review}

\section{Conclusion}

Data reviewed showed the need for further research to establish universal nutrition strategies on healthy dietary intake to improve women's health and pregnancy outcome. Studies showed healthy dietary patterns reduced high blood pressure, pregnancy induced hypertension, excessive weight gain, anaemia and improved weight gain in underweight pregnant women while energy-food diets increased health risk in pregnancy and birth complications. This systematic review revealed the relevance to adopt dietary management and nutrition strategies in clinical practices as public health concern. A Cochrane database systematic review suggested that dietary patterns implementation in clinical management of pregnant women is essential. ${ }^{11}$ 


\section{Effect of Dietary Patterns and, or Nutrition Strategies Reducing Risk in Adverse Maternal Health Occurrences during Pregnancy: A Systematic Review}

\section{Reference}

1. World Health Organization, 2016. WHO Recommendations on Antenatal Care for a Positive Pregnancy Experience. WHO/RHR/16.12. WHO website (http://www.who.int)

2. Fowles ER \& Marcena Gabrielson M, 2010. First Trimester Predictors of Diet and Birth Outcomes in Low-Income Pregnant Women. Journal of Community Health Nursing. Vol., 22, Pges 117-130. PubMed.

3. Zuccolotto DCC, Crivellent CL, Laércio JF, Sartorelli DS, 2019. Dietary patterns of pregnant women, maternal excessive body weight and gestational diabetes. Vol.53:52. Google Scholar

4. Safaa S, Eshra D, Saleem N., 2016. Effect of malnutrition during pregnancy on pregnancy outcomes. $18^{\text {th }}$ International Conference on Nursing \& Healthcare. J Nurs Care 5:10 (Suppl). Google Scholar

5. Schwingshackl L, Chaimani A, Hoffmann G, Schwedhelm C, Boeing H., 2017. Impact of different dietary approaches on blood pressure in hypertensive and prehypertensive patients: protocol for a systematic review and network meta-analysis. BMJ Open. 2017 Apr 26;7(4):e014736. doi: 10.1136/bmjopen-2016-014736. PubMed

6. Schoenaker DAJM, Sabita S, Soedamah-Muthu, Mishra GD, 2014. The association between dietary factors and gestational hypertension and pre-eclampsia: observational studies. School of Population Health, University of Queensland, Brisbane, Queensland Australia. PubMed.

7. Piirainen T, Isolauri E, Lagström H, Laitinen K, (2006). Impact of dietary counselling on nutrient intake during pregnancy: a prospective cohort study. Br J Nutr. 96(6):1095-104. doi: 10.1017/bjn20061952. PubMed

8. Braunthal S, \& Brateanu A, 2019. Hypertension in pregnancy: Pathophysiology and treatment. SAGE Open Med. 10;7: 2050312119843700. doi: 10.1177/2050312119843700. PubMed.

9. Brown MA, Magee LA, Kenny LC, Ananth KS, McCarthy FP, Shigeru S, Hall DR, Warren CE, Adoyi G, Salisu I, 2018. Hypertensive Disorders of Pregnancy: Classification, Diagnosis, and Management. American Heart Association, Inc Hypertension. 2018; 72:2443. http://hyper.ahajournals.org PubMed

10. VanderJagt DJ, Brock HS, Melah GS, El-Nafaty AU, Crossey MJ, Glew RH, 2007. Nutritional Factors Associated with Anaemia in Pregnant Women in Northern Nigeria. J Health Popul Nutr. 2007 Mar; 25(1): 75-81. PubMed

11. Kibret KT, Chojenta C, Gresham E, Tegegne TK, 2018. Maternal dietary patterns and risk of adverse pregnancy (hypertensive disorders of pregnancy and gestational diabetes mellitus) and birth (preterm birth and low birth weight) outcomes: a systematic review and meta-analysis. Public Health Nutrition: Volume 22, Issue 3, pp. 506-520. Cambridge University Press.

12. Pomerleau J, Lock J, Knai C, McKee M., 2005. Interventions Designed to Increase Adult Fruit and Vegetable Intake Can Be Effective: A Systematic Review of the Literature. The 
Effect of Dietary Patterns and, or Nutrition Strategies Reducing Risk in Adverse Maternal Health Occurrences during Pregnancy: A Systematic Review

Journal of Nutrition, Volume 135(10): 2486-2495, https://doi.org/10.1093/jn/135.10.2486. Oxford Academic

13. Pem D, \& Jeewon R, 2015. Fruit and Vegetable Intake: Benefits and Progress of Nutrition Education Interventions- Narrative Review Article. Iran J Public Health. 44(10): 13091321. PubMed

14. World Health Organization, 2013. Essential Nutrition Actions: Improving Maternal, Newborn, Infant and Young Child Health and Nutrition. Geneva. PubMed

15. Sunuwar DR, Sangroula RK, Shakya NS, Yadav R, Chaudhary NK, Pradhan PMS, 2019. Effect of nutrition education on hemoglobin level in pregnant women: A quasiexperimental study. PLoS ONE 14(3): journal.pone. Google Scholar

16. Abubakari A, Jahn A, 2016. Maternal Dietary Patterns and Practices and Birth Weight in Northern Ghana. PLoS ONE 11(9): journal.pone.0162285. Google Scholar

17. Wrottesley SV, Pisa PT, Shane A. Norris SA, 2017. The Influence of Maternal Dietary Patterns on Body Mass Index and Gestational Weight Gain in Urban Black South African Women. Nutrients; 9(7): 732. PubMed

18. Salam RA, Syed B, Syed S, Das JD, Zagre NM, Rayco-Solon P, Zulfiqar AB., 2015. Maternal nutrition: how is Eastern and Southern Africa faring and what needs to be done? Afr Health Sci. 15(2): 532-545. PubMed

19. Gay HC, Rao SG, Vaccarino V, Ali MK, 2016. Effects of Different Dietary Interventions on Blood Pressure: Systematic Review and Meta-Analysis of Randomized Controlled Trials. Hypertension. 67(4):733-9.Epub

20. Ikem E, Halldorsson TI, Birgisdóttir BE, Rasmussen MA, Olsen SF, MaslovaE, 2019. Dietary Patterns and the Risk of Pregnancy-Associated Hypertension in the Danish National Birth Cohort: A Prospective Longitudinal Study. BJOG, 126 (5), 663-673 PubMed

21. Ramkripa R, Dreibelbis C, Kingshipp BL, Yat Ping Wong, Abrams B, Alison DG, Rasmussen KM, Siega-Riz AM, Stang J, Casavale KO, Spahn JM, Stood EE, 2019.Dietary Patterns Before and During Pregnancy and Maternal Outcomes: A Systematic Review. Am J Clin Nutr, 109 (Suppl_7), 705S-728S Google

22. Jarman M, Mathe N, Ramazani F, Pakseresht M, Robson PJ, Johnson ST, Bell RC, 2018. Dietary Patterns Prior to Pregnancy and Associations with Pregnancy Complications. Nutrients,10(7), 914. doi:10.3390/nu10070914. MDPI

23. Brantsaeter AL, Haugen M, Samuelsen SO, Torjusen H, Trogstad L, Alexander J, Magnus $\mathrm{P}$, Meltzer HM, 2009. A dietary pattern characterized by high intake of vegetables, fruits, and vegetable oils is associated with reduced risk of preeclampsia in nulliparous pregnant Norwegian women. J Nutr. 139(6):1162-8. Epub

24. Schoenaker DAJM, Sabita S, Soedamah-Muthu, Callaway LK, Mishra GD, 2015. Prepregnancy Dietary Patterns and Risk of Developing Hypertensive Disorders of Pregnancy: Results From the Australian Longitudinal Study on Women's Health. Am J Clin Nutr 102 (1), 94-101. PMID 
Effect of Dietary Patterns and, or Nutrition Strategies Reducing Risk in Adverse Maternal Health Occurrences during Pregnancy: A Systematic Review

25. Schoenaker DAJM, Sabita S, Soedamah-Muthu, Mishra GD, 2016. Quantifying the Mediating Effect of Body Mass Index on the Relation between a Mediterranean Diet and Development of Maternal Pregnancy Complications: The Australian Longitudinal Study on Women's Health. Am J Clin Nutr, 104 (3), 638-45 PubMed

26. Timmermans S, Steegers-Theunissen RPM, Vujkovic M, Bakker R, den Breeijen H, Raat H, Russcher H, Lindemans J, Hofman A, Jaddoe VW, Steegers EA, 2011, 2012. Major dietary patterns and blood pressure patterns during pregnancy: the Generation R Study. Am J Obstet Gynecol. 205(4): 337. Goggle Scholar, Epub

27. Demilew YM, Alene GD, Belachew T, 2020. Effect of guided counseling on nutritional status of pregnant women in West Gojjam zone, Ethiopia: a cluster-randomized controlled trial. Nutr J. 19: 38. PubMed

28. Fulay AP, Rifas-Shiman, SL, Oken E., 2018. Associations of the dietary approaches to stop hypertension (DASH) diet with pregnancy complications in Project Viva. Eur J Clin Nutr 72, 1385-1395. https://doi.org/10.1038/s41430-017-0068-8. Google Scholar.

29. Goodarzi-Khoigani M, Baghiani Moghadam MH, Nadjarzadeh A, Mardanian F, Fallahzadeh H, Mazloomy-Mahmoodabad S., 2018. Impact of Nutrition Education in Improving Dietary Pattern During Pregnancy Based on Pender's Health Promotion Model: 23(1): 18-25. IJNMR_198_16, PMCID.

30. Abdel-Aziz SB, Hegazy IS, Mohamed DA, Abu MMA, Kasem EL, Hagag SS, 2018. Effect of dietary counseling on preventing excessive weight gain during pregnancy. Volume 154, Pg 172-181. ScienceDirect.

31. Girard AW \& Olude O, 2012. Nutrition Education and Counselling Provided during Pregnancy: Effects on Maternal, Neonatal and Child Health Outcomes. Paediatr Perinat Epidemiol:26 (Suppl. 1), 191-204. PubMed.

32. Garg A, \& Kashyap S., 2006. Effect of counseling on Nutritional status during pregnancy. Indian J Pediatr 73(8), pp 687-692. https://doi.org/10.1007/BF02898446. Springer link

33. Martin CL, Sotres-Alvarez D, Siega-Riz AM, 2015. Maternal Dietary Patterns during the Second Trimester Are Associated with Preterm Birth. J Nutr.145 (8): 1857-64. doi: 10.3945/jn.115.212019 Epub

34. World Health Organization, 2018. Nutrition: Releases Tips for Healthy Diet. Google

35. Stang J, \& Huffman LG, (2016). Position of the Academy of Nutrition and Dietetics: Obesity, Reproduction, and Pregnancy Outcomes. J Acad Nutr Diet, 116 (4), 677-91. PubMed

36. Chen X, Zhao D, Mao X, Xia Y, Baker PN, Hua Zhang H, 2016. Maternal Dietary Patterns and Pregnancy Outcome. Nutrients; 8(6): 351. pii: nu8060351. doi: 10.3390/nu8060351. PubMed. 


\section{Data Extraction and Selection Process}

\begin{tabular}{|c|c|}
\hline Searched Articles=25 & \\
\hline $\begin{array}{l}\text { Eighteen eligible Articles Screened from Goggle and } \\
\text { PubMed: } \\
\text { Full text=12, Abstracts }=4 \text {, Systematic reviewed articles=2 }\end{array}$ & $\begin{array}{c}\text { Reiected Articles: } \\
\text { Non eligibility Google }=4 ; \text { PubMed }=3\end{array}$ \\
\hline & Relevant Articles \\
\hline $\begin{array}{l}\text { Inclusion criteria: } \\
\text { Pregnant women with gestational hypertension/high blood } \\
\text { pressure, increased BMI/overweight, anaemia, underweight } \\
\text { or malnourished put on dietary patterns, nutrition education } \\
\text { or counseling. } \\
\text { Dietary patterns: Mediterranean only=2; DASH diet } \\
\text { only=1, Healthy diet only=1, Mediterranean vs Traditional } \\
\text { diet=2, dietary counseling + DASH vs Western diet or } \\
\text { Healthy diet vs high-energy food= } 2 \text {, Nutrition Counseling } \\
+ \text { advice +healthy diet=1, Sea food +fish + vegetables }=1 \text {, } \\
\text { Healthy diet vs Western/Tradition/high-energy food= } 2 \text {; } \\
\text { Nutrition strategies + healthy diets + KAP= } 2 \text {, Nutrition } \\
\text { strategies only=4. } \\
\text { Study design: randomize control trial, prospective cohort; } \\
\text { observational/longitudinal }\end{array}$ & $\begin{array}{l}\text { Exclusion: conditions with chronic } \\
\text { hypertension, eclampsia = } 3 \\
\text { Rejected duplicates: dietary pattern with } \\
\text { hypertension-diabetes during pregnancy or } \\
\text { food supplements }=4\end{array}$ \\
\hline
\end{tabular}

Figure 1

Flow chart showing screening process for eligible and non-eligible articles. 